\title{
A Large-Scale Observational Study on the Temporal Trends and Risk Factors of Opioid Overdose: Real-World Evidence for Better Opioids
}

\author{
Jianyuan Deng ${ }^{1} \cdot$ Wei Hou $^{2} \cdot$ Xinyu Dong ${ }^{3} \cdot$ Janos Hajagos $^{1} \cdot$ Mary Saltz $^{1} \cdot$ Joel Saltz $^{1} \cdot$ Fusheng Wang $^{1,3}$ (D)
}

Accepted: 22 April 2021 / Published online: 26 May 2021

(c) The Author(s) 2021

\begin{abstract}
Background The USA is in the midst of an opioid overdose epidemic. To address the epidemic, we conducted a large-scale population study on opioid overdose.

Objectives The primary objective of this study was to evaluate the temporal trends and risk factors of inpatient opioid overdose. Based on its patterns, the secondary objective was to examine the innate properties of opioid analgesics underlying reduced overdose effects.

Methods A retrospective cross-sectional study was conducted based on a large-scale inpatient electronic health records database, Cerner Health Facts ${ }^{\circledR}$, with (1) inclusion criteria for participants as patients admitted between 1 January, 2009 and 31 December, 2017 and (2) measurements as opioid overdose prevalence by year, demographics, and prescription opioid exposures.

Results A total of 4,720,041 patients with 7,339,480 inpatient encounters were retrieved from Cerner Health Facts ${ }^{\circledR}$. Among them, $30.2 \%$ patients were aged $65+$ years, $57.0 \%$ female, $70.1 \%$ Caucasian, $42.3 \%$ single, $32.0 \%$ from the South, and $80.8 \%$ in an urban area. From 2009 to 2017, annual opioid overdose prevalence per 1000 patients significantly increased from 3.7 to 11.9 with an adjusted odds ratio (aOR): $1.16,95 \%$ confidence interval (CI) 1.15-1.16. Compared to the major demographic counterparts, being in (1) age group: 41-50 years (overall aOR 1.36, 95\% CI 1.31-1.40) or 51-64 years (overall aOR 1.35, 95\% CI 1.32-1.39), (2) marital status: divorced (overall aOR 1.19, 95\% CI 1.15-1.23), and (3) census region: West (overall aOR $1.32,95 \%$ CI 1.28-1.36) were significantly associated with a higher odds of opioid overdose. Prescription opioid exposures were also associated with an increased odds of opioid overdose, such as meperidine (overall aOR 1.09, 95\% CI 1.06-1.13) and tramadol (overall aOR 2.20,95\% CI 2.14-2.27). Examination on the relationships between opioid analgesic properties and their association strengths, aORs, and opioid overdose showed that lower aOR values were significantly associated with (1) high molecular weight, (2) non-interaction with multi-drug resistance protein 1 or interaction with cytochrome P450 3A4, and (3) non-interaction with the delta opioid receptor or kappa opioid receptor.

Conclusions The significant increasing trends of opioid overdose at the inpatient care setting from 2009 to 2017 suggested an ongoing need for efforts to combat the opioid overdose epidemic in the USA. Risk factors associated with opioid overdose included patient demographics and prescription opioid exposures. Moreover, there are physicochemical, pharmacokinetic, and pharmacodynamic properties underlying reduced overdose effects, which can be utilized to develop better opioids.
\end{abstract}

Fusheng Wang

fusheng.wang@stonybrook.edu

1 Department of Biomedical Informatics, Stony Brook University, 2313D Computer Science, Stony Brook, NY 11794-8330, USA

2 Department of Family, Population and Preventive Medicine, Stony Brook University, Stony Brook, NY, USA

3 Department of Computer Science, Stony Brook University, Stony Brook, NY, USA 


\section{Key Points}

There were significant increasing trends of opioid overdose at the US inpatient care setting from 2009 to 2017, showing an ongoing need for opioid overdose prevention

Different prescription opioids were associated with opioid overdose to different extents, indicating a necessity to differentiate prescription opioids during prescribing

The optimal properties underlying reduced overdose effects mined from the large-scale, real-world electronic health records hold significant potential to guide the development of better opioids with reduced overdose effects

\section{Introduction}

Over the past two decades, drug overdose has been a leading cause of injury-related deaths in the USA, of which 70\% involved illicit or prescription opioids [1]. Prescription opioids are among the most effective drugs to treat pain, which are ligands to the endogenous mu opioid receptor (MOR) and can exert agonistic, partially agonistic, or antagonistic effects [2]. When activated by agonists, MOR can mediate analgesic effects as well as modulate respiratory responses [3]. However, lethal respiratory depression can happen when overdosed [3]. In 2018, opioid overdose was attributed to 47,761 deaths, which imposed an enormous public health burden on the USA $[4,5]$. The epidemic of opioid overdose is dynamic and complex [6,7]. As opined by Jalal et al. [7], the current epidemic can be a recent manifestation of an ongoing longer term process. Close monitoring of the temporal trends of opioid overdose is, therefore, crucial for developing and evaluating relevant policies to prevent and control the epidemic [7-11]. Furthermore, despite the complexity of the opioid overdose epidemic, there exist some patterns between opioid overdose and factors such as patient demographics and opioid prescriptions, which can provide essential knowledge for the planning of effective prevention measures [11-14].

To combat the opioid overdose epidemic, various guidelines and programs have already been launched, mainly to curb opioid prescribing [15-17]. For example, the Centers for Disease Control and Prevention has recommended various dosage thresholds in morphine milligram equivalents to limit opioid use [17]. Notwithstanding these measures, opioid analgesics are still among the most commonly prescribed medications, presumably because pain is one of the most common reasons for patients to visit their doctors [18, 19]. In addition, to control the quantity of opioid use, another major pursuit of the opioid research community has been developing opioid analgesics with reduced overdose effects [20-26]. More specifically, reduced overdose effects can be potentially addressed from (1) the pharmacokinetic (PK) perspective-what the body does to the drug: decrease the probability of overdose events by avoiding excessive amount of opioids at the action site and (2) the pharmacodynamic (PD) perspective-what the drug does to the body: alleviate the overdose outcome by avoiding unexpected off-target effects. Based on these mechanisms, novel therapeutic agents have been proposed via optimizing certain innate properties of opioid analgesics. For example, one selective MOR agonist was shown to be both effective for analgesia and devoid of respiratory depression [22]. Another MOR agonist was also reported to have low abuse potential because of a reduced rate of entry across the blood-brain barrier (BBB) [27]. Nonetheless, whether these new chemical entities will eventually work in human subjects remains unclear [25]. In fact, new drug development is well known for its low success rate [28]. Given the severity and urgency of the opioid overdose epidemic, early-stage optimization strategies on the lead compounds are crucial for the timely development of better opioids, which involve both PK and PD perspectives [29].

Recently, real-world data, such as electronic health records (EHRs), have received substantial attention for large-scale drug safety studies [30]. Real-world evidence generated from real-world data holds significant potential to guide drug discovery and subsequent drug development [31, 32]. In the case of opioid overdose, the fundamental question would be "What are the optimal properties underlying opioids with reduced overdose effects?" The properties associated with reduced overdose effects in a large-scale human population can be used to generate pertinent hypotheses during early drug discovery as potential optimization strategies [33].

Hence, in this observational study on opioid overdose, we first evaluated the temporal trends of opioid overdose and its risk factors, including patient demographics and prescription opioid exposures based on a real-world EHR database. Second, based on the opioid overdose patterns emerged from the large-scale human population, we examined the opioid analgesic properties underlying lower association strengths in opioid overdose.

\section{Methods}

\subsection{Data and Measurements}

This retrospective cross-sectional study was based on Cerner Health Facts ${ }^{\circledR}$, one of the largest EHR databases in the USA. Health Facts ${ }^{\circledR}$ stores real-world, de-identified, longitudinal 
patient data from over 600 healthcare facilities. Health Facts ${ }^{\circledR}$ is a relational database with multiple tables on patient demographics, encounters, diagnoses, medications, clinical events, procedures, and laboratory tests, among others. The records in Health Facts ${ }^{\circledR}$ are comprehensive and include over 300 data elements. The care settings in Health Facts ${ }^{\circledR}$ include inpatient, outpatient, and emergency, among others. However, only inpatient data in Health Facts ${ }^{\circledR}$ were evaluated against another well-established inpatient sample, the Healthcare Cost and Utilization Project Nationwide Inpatient Sample, which are shown to have a similar distribution across all data elements in a previous study [34]. As pointed out by the same study [34], many hospitals contributing to the Health Facts ${ }^{\circledR}$ database have both paper-based records and electronic records. As a result, there may be missing information in this EHR database. In this study, we only focused on the validated inpatient data.

For the assembly of our study dataset, we applied the inclusion criteria: patients admitted to the inpatient care setting between 1 January, 2009 and 31 December, 2017. No exclusion criteria were applied. For each patient, we aggregated the diagnoses codes from all inpatient encounters within one calendar year. The International Classification of Diseases (ICD) codes for (1) poisoning and (2) adverse effect by opium, heroin, methadone, and other related narcotics, among others, were then used to identify patients with opioid overdose [35, 36]. All relevant ICD Ninth Revision (ICD-9) and Tenth Revision (ICD-10) codes were summarized in Table S1 of the Electronic Supplementary Material (ESM) [37]. Patients with at least one opioid overdose diagnosis code were added to the opioid overdose cohort in that year. Other independent variables are described as follows.

\subsubsection{Patient Demographics}

We measured patient demographics, including age group ( $<18,18-30,31-40,41-50,51-64,65+$ years), sex (female, male), race/ethnicity (African American, Asian, Caucasian, Hispanic, Native American, others), marital status (divorced, married, single, widowed, others), census region (Midwest, Northeast, South, West), and urbanicity (rural, urban). For patients with varied demographics within a calendar year, such as migration between rural and urban areas, records from their first encounter were used.

\subsubsection{Prescription Opioid Exposures}

For each patient, we aggregated the prescribed medications during hospitalization in Health Facts ${ }^{\circledR}$ from all inpatient encounters within one calendar year. A patient was defined as exposed if the aggregated medications containing the active opioid ingredient(s), otherwise as non-exposed. The prescription opioids were further categorized by their action types on MOR [2], namely, (1) opioid agonists: codeine, fentanyl, and morphine, among others, (2) opioid partial agonists: buprenorphine, butorphanol, and nalbuphine, and (3) opioid antagonist: naloxone. For each category, we collected the corresponding US Food and Drug Administrationapproved active ingredients from DrugBank 5.1.6, which were then mapped to medications containing them in Health Facts ${ }^{\circledR}$ [38]. Illicit opioids and opioids withdrawn from the market were excluded, such as diamorphine, i.e., heroin and propoxyphene. To ensure sufficient statistical significance, prescription opioids with annual prevalence (per 1000 patients) less than $1 \%$ were also excluded from analyses, such as dihydrocodeine, naltrexone, and pentazocine.

For the innate properties of prescription opioids, we identified the PK and PD properties reported to be relevant in opioid overdose, including (1) PK properties, interaction with certain efflux transporters and metabolic enzymes: multi-drug resistance protein 1 (MDR1), cytochrome P450 (CYP) 2D6, and CYP3A4 [39-41] and (2) PD properties, interaction with certain receptors: delta opioid receptor (DOR), kappa opioid receptor (KOR), and N-methyl-Daspartate receptor (NMDAR) [22, 42]. In addition to the discrete PK and PD properties, several continuous physicochemical properties underlying BBB permeability were also included: lipophilicity $(\log \mathrm{P})$, polar surface area (PSA), and molecular weight (MW), which can affect the opioid exposures inside the brain [43]. All the properties are commonly seen in the optimization strategies during drug discovery and development [44] and their values were also collected from DrugBank 5.1.6 [38].

\subsection{Statistical Analyses}

Demographic characteristics were determined using descriptive analyses. Proportions of opioid overdose, i.e., opioid overdose prevalence, among all patients and demographic subgroups, were calculated on a yearly basis. Inferential analyses were conducted to examine the temporal trends of opioid overdose and risk factors by calculating the adjusted odds ratios (aORs) and 95\% confidence intervals (CIs). First, for temporal trends in all patients, multivariable logistic regression was used with (1) whether a patient was diagnosed as opioid overdose within a year as the dependent variable and (2) year and demographic factors as independent variables. For each patient subgroup stratified by demographics, the same model was applied with an additional interaction item between the year and the demographic factor to examine the temporal trends, further adjusted for all demographics (e.g., age group, sex, race/ethnicity, marital status, census region, and rural/urban area). Second, for risk factors, including demographics and prescription opioid exposures, multivariable logistic regression adjusted for all 
demographics was also performed based on both data in each year for the yearly aORs and data across all years for the overall aORs. For demographics, age group in $65+$ years, sex as female, race/ethnicity as Caucasian, marital status as single, and census region in the South and urban areas were set as the reference. Note that when evaluating prescription opioid exposures as risk factors, the non-exposed patients were set as the reference.

After calculating the aORs of prescription opioid exposures, we conducted a further analysis for our secondary study objective. To examine the relationship between opioid analgesic innate properties and their aORs (i.e., association strengths) in opioid overdose (exposed patients vs non-exposed patients), a power analysis was first conducted to only exploit aORs with statistical power greater than $80 \%$ $[45,46]$. For continuous physicochemical properties, we discretized them into low or high, with its median value as the cut-off. The opioid analgesics were grouped based on the discrete physicochemical, PK, and PD properties. Then, the Wilcoxon test was used to examine whether aORs in certain property groups were significantly lower, which may correspond to better opioid analgesics with reduced overdose effects.

The dataset was processed in Python 3.7.3. Statistical analyses were conducted in R 3.6.1, packages: "stats" and "epiR". All data analyses were performed between 1 February, 2020 and 1 September, 2020.

\section{Results}

\subsection{Patient Characteristics}

Between 2009 and 2017, a total of 7,339,480 inpatient encounters for 4,720,041 patients (with/without opioid overdose) were retrieved from Health Facts ${ }^{\circledR}$. Among them, $1,423,744(30.2 \%)$ patients were in the $65+$ years age group, $2,689,898(57.0 \%)$ patients were female, 3,310,471 (70.1\%) patients were Caucasian, 1,995,380 patients (42.3\%) were single, 1,511,746 patients (32.0\%) were from the South, and $3,813,817$ patients $(80.8 \%)$ were in an urban area. Encounter counts for patients are summarized in Fig. S1 of the ESM.

\subsection{Temporal Trends of Opioid Overdose}

Annual opioid overdose prevalence from 2009 to 2017 stratified by demographics is detailed in Table 1 . There were significant increasing trends of opioid overdose. From 2009 to 2017 , the overall opioid overdose prevalence per 1000 patients increased from 3.7 to $11.9 \%$ (3.2-fold increase; aOR: $1.16,95 \%$ CI $1.15-1.16 ; p<0.0001)$. The increasing trends in all patient subgroups were also significant. Among them, a patient subgroup from the South had a 6.3-fold increase (from 2.0 to $12.9 \%$; aOR: $1.22,95 \% \mathrm{CI}$ $1.20-1.22 ; p<0.0001$ ), followed by Asian patients (5.2-fold increase: from 1.2 to $6.0 \%$; aOR: $1.20,95 \%$ CI $1.14-1.27$; $p<0.0001)$, African American patients (4.8-fold increase: from 2.2 to $10.8 \%$; aOR: $1.20,95 \%$ CI $1.19-1.22$; $p<$ $0.0001)$, and patients in a rural area (4.2-fold increase: from 2.1 to $8.8 \%$; aOR: $1.14,95 \%$ CI $1.13-1.16 ; p<0.0001$ ). These prominent trends suggested that prevention and control measures at the US inpatient care setting had been inadequate over the years.

\subsection{Demographics as Risk Factors for Opioid Overdose}

Demographics as risk factors for opioid overdose are summarized in Table 2. Compared to patients aged 65+ years, patients aged $41-50$ years (overall aOR: $1.36,95 \% \mathrm{CI}$ $1.31-1.40 ; p<0.0001$ ) or $51-64$ years (overall aOR: 1.35 , 95\% CI 1.32-1.39; $p<0.0001$ ) had a higher odds of opioid overdose whereas patients aged $<18$ years (overall aOR: $0.18,95 \%$ CI $0.17-0.19 ; p<0.0001$ ) or $18-30$ years (overall aOR: $0.74,95 \%$ CI $0.71-0.76 ; p<0.0001$ ) had a much lower odds. When comparing to single patients, patients who were divorced (overall aOR: $1.19,95 \%$ CI $1.15-1.23 ; p<0.0001$ ) had a higher odds of opioid overdose whereas the odds were lower for patients who were married (overall aOR: 0.73, 95\% CI $0.71-0.75 ; p<0.0001$ ) or widowed (overall aOR: 0.89, 95\% CI $0.86-0.93 ; p<0.0001)$. For patients in the rural area (overall aOR: 0.84, 95\% CI 0.82-0.86; $p<0.0001$ ), their odds of opioid overdose were lower than patients in the urban area. Note that the yearly aORs were also varied. For example, across all years, male patients (overall aOR: 0.98, 95\% CI 0.96-1.00; $p=0.05$ ) had comparable odds with female patients. However, male patients were more likely to have opioid overdose in 2009 whereas they became comparably or less likely to have it in subsequent years. Similarly, patients from Midwest, Northeast, or West census regions also exhibited varying aORs of opioid overdose from 2009 to 2017 , which partially reflected the changing dynamics of the opioid overdose epidemic [7].

\subsection{Prescription Opioid Exposures as Risk Factors for Opioid Overdose}

Prescription opioids included for evaluation are listed in Table 3, along with their relevant properties and the overall prevalence per 1000 patients between 2009 and 2017. Prescription opioid exposures as risk factors for opioid overdose are detailed in Table 4. Among MOR agonists, morphine (342.1\%o) was most frequently prescribed, followed by fentanyl (297.5\%o), oxycodone (263.3\%o), hydromorphone ( $242.1 \%$ ) , and hydrocodone (182.4\%o), the high usage rates of which manifested the prescription opioid abuse [47]. 


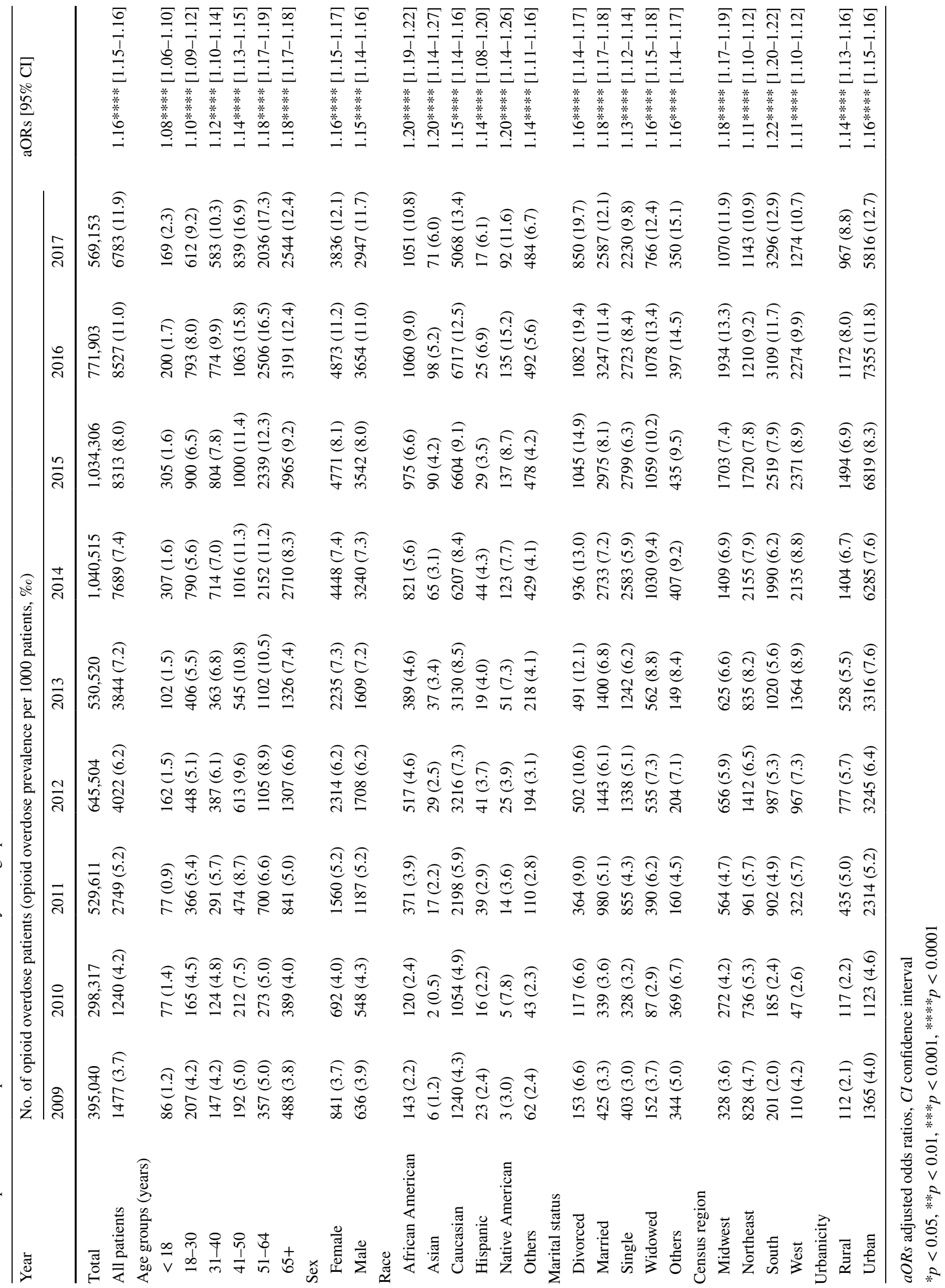




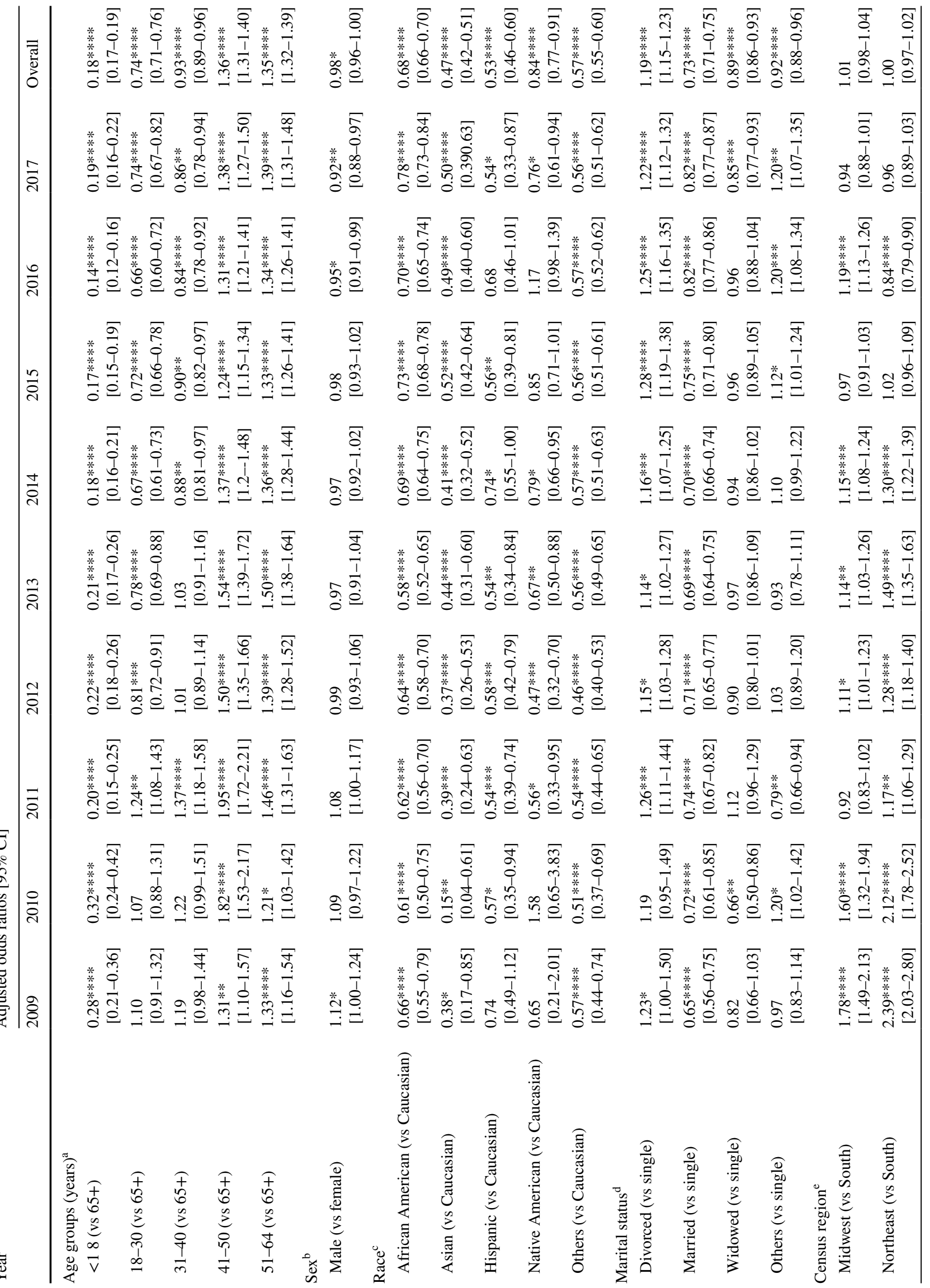




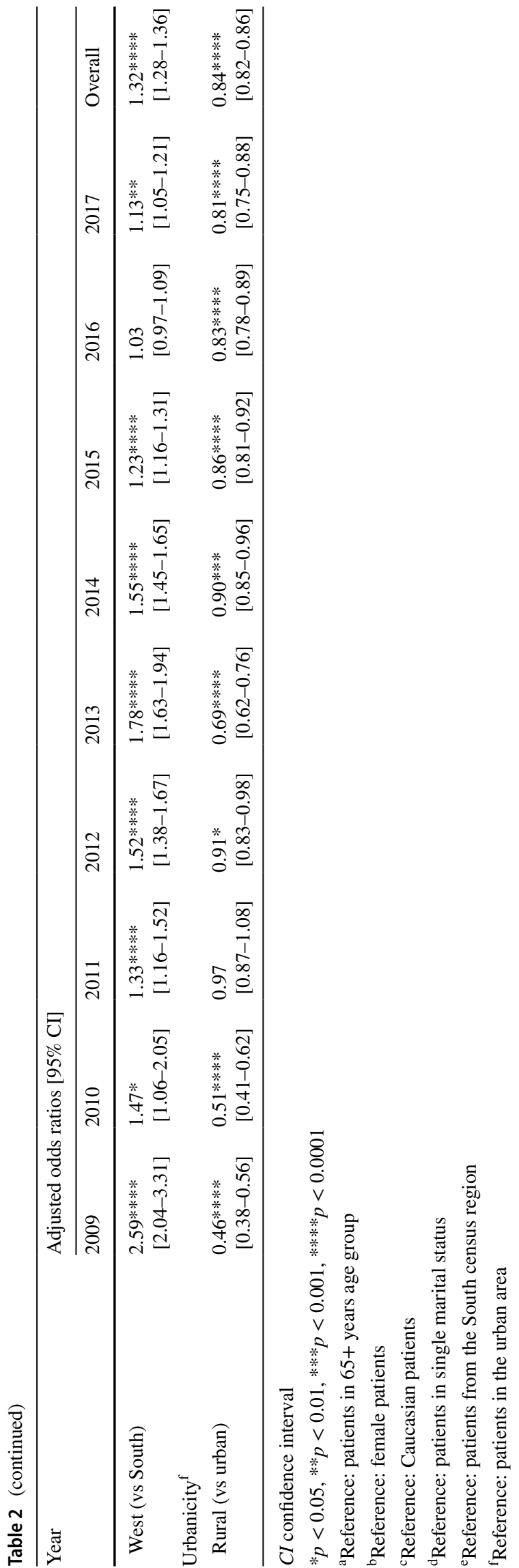

The MOR antagonist, naloxone (157.8\%o), was also commonly prescribed and compared to the non-exposed patients, patients exposed to naloxone (overall aOR: $3.24,95 \% \mathrm{CI}$ $3.18-3.31 ; p<0.0001)$ had a higher odds of opioid overdose, presumably owing to its therapeutic effect to reverse overdose [3].

For MOR partial agonists, buprenorphine (3.2\%o) was less frequently prescribed than nalbuphine $(45.7 \% o$ ) and butorphanol (29.5\%o). In addition, exposures to partial agonists, nalbuphine (overall aOR: 0.565, 95\% CI 0.53-0.60; $p$ $<0.0001$ ) and butorphanol (aOR: 0.09, 95\% CI 0.07-0.11; $p<0.0001$ ) were associated with a reduced odds of overdose whereas buprenorphine (overall aOR: $2.89,95 \%$ CI 2.63-3.19; $p<0.0001$ ) was positively associated with opioid overdose. This could be explained by the fact that buprenorphine is for medication-assisted treatment, which is closely related to opioid overdose [48]. Exposures to opioid agonists were also positively associated with opioid overdose except for codeine (overall aOR: $0.78,95 \%$ CI $0.74-0.83 ; p$ $<0.0001$ ), consistent with a previous finding that codeine had the lowest risk for severe respiratory depression [12]. Moreover, different opioid agonists posed unique aORs, reflecting varied association strengths in opioid overdose.

\subsection{Optimal Properties for Better Opioid Agonists}

To explore the optimal properties underlying opioid analgesics with reduced overdose effects, we examined the relationships between opioid agonist properties (Table 3) and the yearly aORs of opioid overdose when comparing the exposed patients to the non-exposed patients (Table 4), depicted in Fig. 1. Note that opioid agonists targeting for peripheral MORs, namely loperamide and diphenoxylate, and methadone for medication-assisted treatment purposes, were excluded in this analysis [48, 49]. For the physicochemical properties, lower aORs, i.e., weakened association strengths, were observed for opioid agonists with high MW (median decreased from 1.62 to $1.24 ; p<0.001$ ). The interpretation is that when an opioid agonist's MW is high, its association with opioid overdose would be weakened, possibly owing to reduced BBB permeability [43]. For lipophilicity (median decreased from 1.49 to $1.40 ; p=0.2$ ) and polar surface area (median increased from 1.25 to $1.60 ; p=$ $0.45)$, no significant associations were observed.

From the PK perspective, a non-interaction with MDR1 was associated with lower aORs (median decreased from 1.51 to $1.25 ; p<0.05$ ), which indicates that if an opioid agonist does not interact with MDR1, its association strength with opioid overdose would be lowered. This could be because of an interaction between opioids and MDR 1 at the BBB that may up-regulate the expression of MDR1, the high expression level of which was reported to be related to opioid tolerance, abuse, dependence, and eventually overdose 
Table 3 Relevant properties of prescription opioids and the overall opioid use prevalence per 1000 patients (\%o) between 2009 and 2017

\begin{tabular}{|c|c|c|c|c|c|c|c|c|c|c|}
\hline \multirow[t]{2}{*}{ Prescription opioids ${ }^{\mathrm{a}}$} & \multicolumn{3}{|c|}{ Physicochemical properties ${ }^{\mathrm{b}}$} & \multicolumn{3}{|c|}{ Pharmacokinetic properties $^{c}$} & \multicolumn{3}{|c|}{ Pharmacodynamic properties ${ }^{\mathrm{d}}$} & \multirow[t]{2}{*}{ Prevalence $(\% \circ$} \\
\hline & $\log \mathrm{P}$ & PSA & MW & MDR1 & CYP2D6 & CYP3A4 & DOR & KOR & NMDAR & \\
\hline \multicolumn{11}{|l|}{ Agonist } \\
\hline Morphine & 1.0 & 52.9 & 285.3 & + & - & + & + & + & - & 342.1 \\
\hline Fentanyl & 4.1 & 23.6 & 336.5 & + & - & + & + & + & - & 297.5 \\
\hline Oxycodone & 1.0 & 59.0 & 315.4 & - & + & + & + & + & - & 263.3 \\
\hline Hydromorphone & 1.7 & 49.8 & 285.3 & - & - & - & + & + & - & 242.1 \\
\hline Hydrocodone & 2.1 & 38.8 & 299.4 & - & + & + & + & - & - & 182.4 \\
\hline Meperidine & 2.9 & 29.5 & 247.3 & - & + & + & - & + & + & 82.3 \\
\hline Tramadol & 2.7 & 32.7 & 263.4 & + & + & + & + & + & + & 55.7 \\
\hline Codeine & 1.2 & 41.9 & 299.4 & - & + & + & + & + & - & 43.9 \\
\hline Loperamide & 4.4 & 43.8 & 477.0 & + & + & + & + & + & - & 23.1 \\
\hline Diphenoxylate & 5.7 & 53.3 & 452.6 & - & - & - & + & - & - & 8.4 \\
\hline Methadone & 4.1 & 20.3 & 309.4 & + & + & + & + & - & + & 7.8 \\
\hline \multicolumn{11}{|l|}{ Partial agonist } \\
\hline Nalbuphine & 2.0 & 73.2 & 357.4 & - & - & - & + & + & - & 45.7 \\
\hline Butorphanol & 3.7 & 43.7 & 327.5 & - & - & - & + & + & - & 29.5 \\
\hline Buprenorphine & 4.5 & 62.2 & 467.6 & + & + & + & + & + & - & 3.2 \\
\hline \multicolumn{11}{|l|}{ Antagonist } \\
\hline Naloxone & 1.5 & 70.0 & 327.4 & - & - & + & + & + & - & 157.8 \\
\hline
\end{tabular}

CYP cytochrome P450, DOR delta opioid receptor, KOR kappa opioid receptor, $\log P$ lipophilicity, $M D R 1$ multi-drug resistance protein 1 , $M W$ molecular weight, NMDAR $N$-methyl-D-aspartate receptor, $P S A$ polar surface area

${ }^{a}$ Prescription opioids are categorized by their action on the mu opioid receptor (MOR), ordered by their overall prevalence

${ }^{\mathrm{b}}$ Relevant physicochemical properties include $\log \mathrm{P}$, PSA, and MW

${ }^{\mathrm{c}}$ Relevant pharmacokinetic properties include whether the opioid interacts with [+ for interacting, - for not interacting] (1) efflux transporter, MDR1 and (2) metabolic enzymes, CYP2D6 and CYP3A4

${ }^{\mathrm{d}}$ Relevant pharmacodynamic properties include whether the opioid interacts with [+ for interacting, - for not interacting] (1) other opioid receptors, DOR and KOR and (2) non-opioid receptor in modulating opioid analgesia

[50, 51]. For CYP3A4, an interaction was associated with lower aORs (median decreased from 1.79 to $1.32 ; p<0.01$ ), which suggests that CYP3A4 metabolism may be a protective factor for opioid agonist overdose as a result of reduced bioavailability during first-pass metabolism [40]. From the PD perspective, lower aORs were observed when opioid agonists do not interact with DOR (median decreased from 1.52 to $1.13 ; p<0.01$ ) or KOR (median decreased from 1.53 to $1.23 ; p=0.07$ ). The interpretation is that when opioid agonists are selective towards MOR, overdose effects would be reduced. For NMDAR (median increased from 1.36 to $1.69 ; p=0.16$ ), no significant difference in aORs was observed.

\section{Discussion}

\subsection{Ongoing Need for Targeted Overdose Prevention}

In this study, we found that opioid overdose increased significantly at the US inpatient care setting from 2009 to 2017. As pointed out by Danovitch et al. [52], inpatient opioid overdose is a serious harm, yet preventable, and is likely to be underestimated in much of the current literature. The significant increasing trends observed in Health Facts ${ }^{\circledR}$, a large-scale EHR database, suggested that prevention and control measures for opioid overdose had been inadequate at the US inpatient setting over these years, especially for patient subgroups with prominent increasing trends, such as patients from the South and patients in a rural area.

We also quantified how patient demographics were associated with inpatient opioid overdose and found that being in certain demographic subgroups increased the odds of opioid overdose. For instance, compared to the 


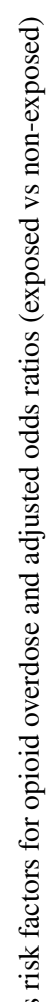

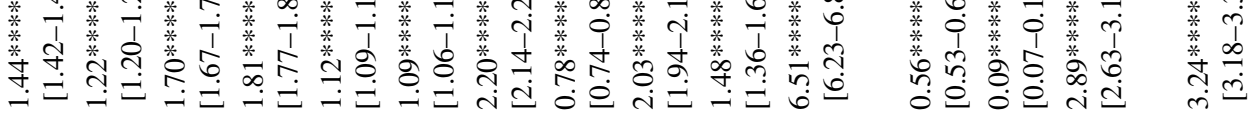

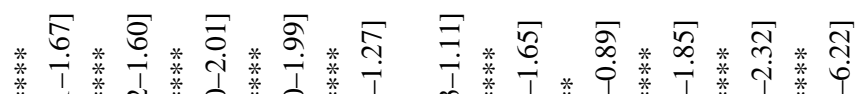

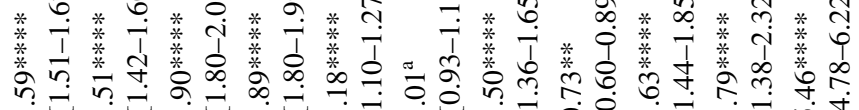

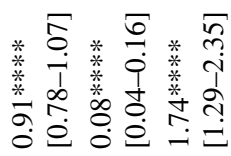

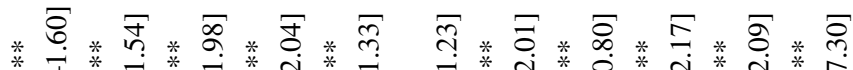

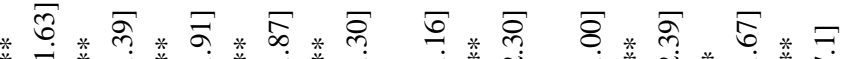

.

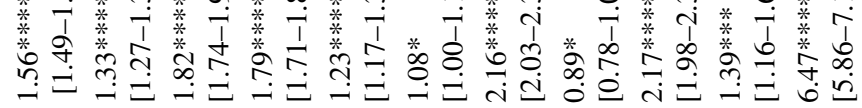

*

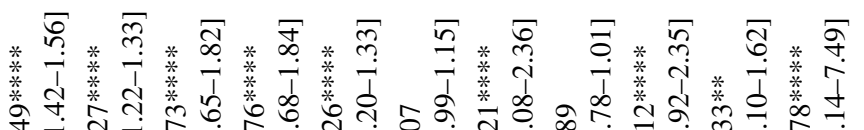

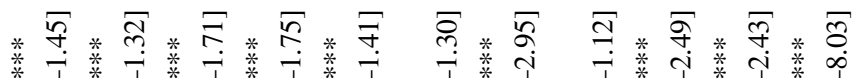

* 守 $\bar{m} * \bar{\infty}$

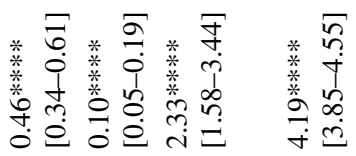



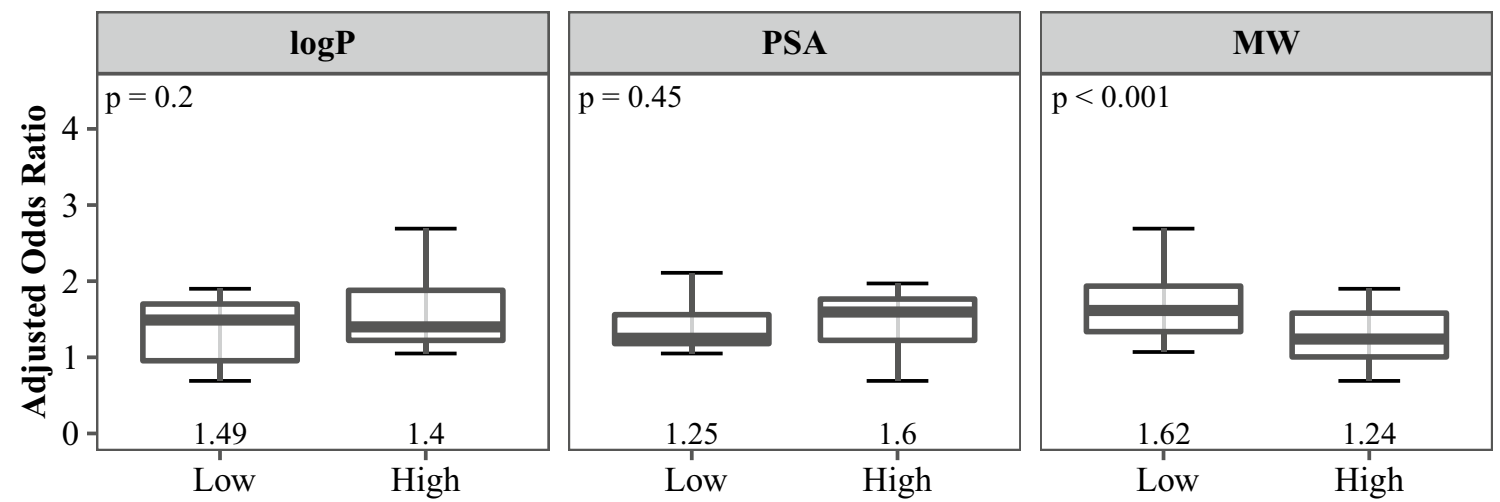

(A) Physicochemical Properties
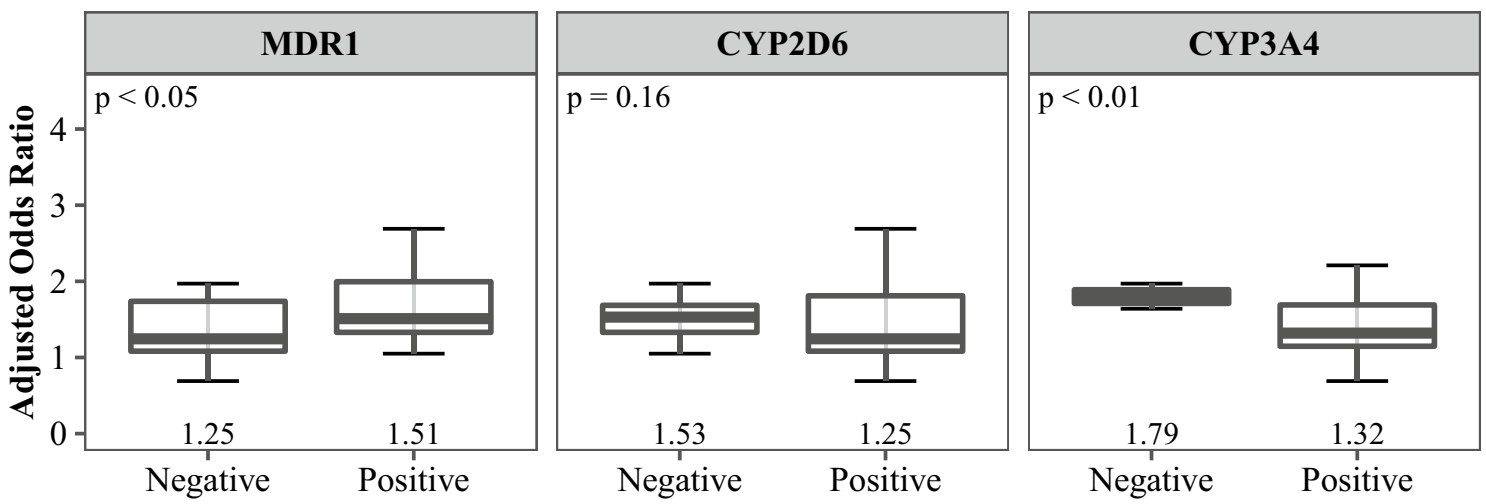

(B) Pharmacokinetic Interaction
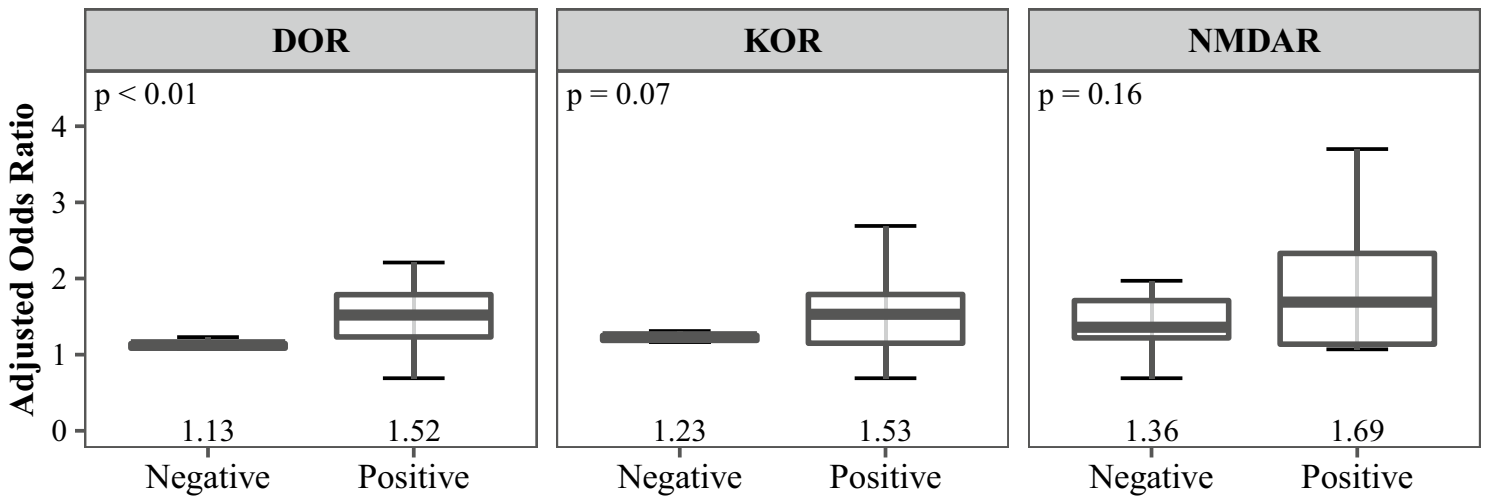

(C) Pharmacodynamic Interaction

Fig. 1 Relationships between innate properties of opioid agonists and the adjusted odds ratios in opioid overdose. a Wilcoxon test on opioid agonist adjusted odds ratios in opioid overdose and their discretized physicochemical properties: lipophilicity $(\log \mathrm{P})$, polar surface area (PSA), and molecular weight (MW). b Wilcoxon test on opioid agonist adjusted odds ratios in opioid overdose and whether they interact with (+ for interacting, - for not interacting) efflux transporter

major demographic counterpart age $65+$ years, patients aged 51-64 and 41-50 years were more likely to have opioid overdose, which was partly consistent with a recent Mortality Disparities in American Communities study, where patients aged 40-59 years were most attributed to opioid overdose deaths from 2008 to 2015 [53]. Patients in and metabolic enzymes: multi-drug resistance protein 1 (MDR1), cytochrome P450 (CYP) 2D6, and CYP3A4. c Wilcoxon test on opioid agonist adjusted odds ratios in opioid overdose and whether they interact with (+ for interacting, - for not interacting) receptors: delta opioid receptor (DOR), kappa opioid receptor (KOR), and $N$-methylD-aspartate receptor (NMDAR)

the urban area had a higher odds of opioid overdose than those in a rural area, which reflected the urbanicity of the opioid overdose epidemic [53]. These associated risk factors suggest an urgent need for targeted measures in patient subgroups at a higher risk. 


\subsection{Necessity to Differentiate Prescription Opioids}

Prescription opioid exposures were also evaluated as risk factors for opioid overdose. Our study showed that prescription opioids were commonly prescribed. For instance, 342.1 per 1000 patients were prescribed medications containing morphine, the high usage rate of which manifested inappropriate opioid prescribing practice and, eventually, prevalent opioid overdose [54]. Previous studies showed that opioid overdose was associated with opioid prescription patterns, such as dosage and duration [36, 55-57]. However, measures to reduce prescription opioid supply alone may not be enough to combat the opioid crisis [58].

In the current literature, prescription opioids were usually aggregated for the morphine milligram equivalent calculation, a widely adopted metric to control the quantity of opioid use [15-17]. One potential misconception arising from morphine milligram equivalents is that all opioids are interchangeable, which may falsely justify the transitioning of one opioid to other "equivalent" opioids $[59,60]$. A previous study based on a cohort of 307 patients showed that some prescription opioids were associated with a much higher risk for severe respiratory depression than others [12]. For example, fentanyl had a 20-fold higher relative risk compared with codeine, the lowest-risk opioid. In fact, a wide range of differences exist among prescription opioids. For instance, during distribution to the site of action, i.e., MOR in the central nervous system, they exhibit varied BBB permeability [61]. In addition, opioids also have disparate binding profiles to the opioid receptors $[62,63]$. Our study revealed varied associations with opioid overdose for different prescription opioids, indicating a necessity to differentiate prescription opioids in opioid prescribing practice [12].

\subsection{Real-World Evidence on Optimal Properties for Better Opioids}

Given the foreseeable continuing trends of opioid overdose in the USA and the high prevalence of prescription opioids for pain, there is an undisputed need to develop better opioid analgesics with reduced overdose effects. Our study generated real-world evidence on potential optimal properties. For instance, from the physicochemical perspective, opioid agonists with low MW had significantly higher association strengths with opioid overdose, possibly owing to increased BBB permeability. From PK and PD perspectives, when opioid agonists interact with MDR1, DOR, or KOR, the association strengths in opioid overdose were significantly increased. Thus, the interactions with the efflux transporter and other opioid receptors should be taken into consideration during early drug discovery for better opioid agonists. Although some optimal properties have been proposed before, they were mostly based on preclinical studies and there still lacks a clear link to the safety profiles in humans. With the emergence of artificial intelligence in drug discovery, molecular structure design biased towards pre-defined attributes has become a reality $[64,65]$. The real-world evidence on the optimal properties from large-scale data may be used to generate pertinent hypotheses for optimization strategies during the development of better opioids [30].

\subsection{Limitations}

Our study also had some limitations. First, we only used ICD-9/ICD-10 codes to identify patients with opioid overdose, which may not be fully accurate $[66,67]$. In addition, there was a transition of ICD-9 codes to ICD-10 codes in 2015, which may lead to discontinuities in the opioid overdose trends [68]. Second, we only focused on patient demographics and prescription opioid exposures as risk factors for opioid overdose. Other potential risk factors such as comorbidities and surgery procedures were not examined [69, 70]. In addition, opioid prescription patterns including dosage and duration also need further assessment. Third, because of the observational study design, where we did not strictly distinguish the temporal order of being prescribed with a medication and getting diagnosed as opioid overdose, the association strengths, thus, may not necessarily represent causality. Last, our study was based on a single database. Exploitation on other real-world data sources would also be needed.

Nevertheless, despite the above limitations, we conducted a comprehensive study on inpatient opioid overdose with the objectives to (1) evaluate its temporal trends and associated risk factors and (2) examine the innate properties of opioid analgesics underlying reduced overdose effects, which provided critical knowledge to address the opioid overdose epidemic.

\section{Conclusions}

A retrospective observational study was conducted using a large-scale EHR database to evaluate opioid overdose patterns, including temporal trends and associated risk factors. The significant increasing trends of opioid overdose from 2009 to 2017 suggested that prevention and control measures for opioid overdose had been inadequate at the US inpatient care setting. In addition, patient demographics and prescription opioid exposures were associated with a higher odds of opioid overdose, suggesting that targeted prevention and control measures are needed. Furthermore, there are optimal properties underlying opioid analgesics with reduced overdose effects, which hold significant potential as optimization strategies for developing better opioids. 
Supplementary Information The online version contains supplementary material available at https://doi.org/10.1007/s40801-021-00253-8.

\section{Declarations}

Funding Jianyuan Deng and Xinyu Dong are funded by the Stony Brook University OVPR Seed Grant 1158484-63845-6.

Conflicts of interest Jianyuan Deng, Wei Hou, Xinyu Dong, Janos Hajagos, Mary Saltz, Joel Saltz, and Fusheng Wang have no conflicts of interest that are directly relevant to the content of this article.

Ethics approval The use of Cerner Health Facts ${ }^{\circledR}$ for research purposes is approved by Cerner and Stony Brook Medicine.

Consent to participate Not applicable.

Consent for publication Not applicable.

Availability of data and material The diagnosis codes for opioid overdose and the encounter counts for patients in Cerner Health Facts $®$ are provided in the ESM.

Code availability Not applicable.

Author contributions JD, WH, XD, and FW conceptualized and designed the study. JD, XD, JH, FW, MS, and JS participated in the data acquisition process. JD and $\mathrm{WH}$ analyzed the data and conducted the statistical analyses. JD, WH, XD, and FW performed the data interpretation. JD drafted the manuscript. FW obtained the funding. FW, JH, MS, and JS provided administrative and technical support. All authors conducted a critical review and revision of the manuscript.

Open Access This article is licensed under a Creative Commons Attribution-NonCommercial 4.0 International License, which permits any non-commercial use, sharing, adaptation, distribution and reproduction in any medium or format, as long as you give appropriate credit to the original author(s) and the source, provide a link to the Creative Commons licence, and indicate if changes were made. The images or other third party material in this article are included in the article's Creative Commons licence, unless indicated otherwise in a credit line to the material. If material is not included in the article's Creative Commons licence and your intended use is not permitted by statutory regulation or exceeds the permitted use, you will need to obtain permission directly from the copyright holder. To view a copy of this licence, visit http://creativecommons.org/licenses/by-nc/4.0/.

\section{References}

1. Hedegaard H, Minino AM, Warner M. Drug overdose deaths in the United States, 1999-2018. NCHS Data Brief. 2020;356:1-8.

2. James A, Williams J. Basic opioid pharmacology: an update. $\mathrm{Br}$ J Pain. 2020;14(2):115-21.

3. Boyer EW. Management of opioid analgesic overdose. New Engl J Med. 2012;367(14):1372-3.

4. Wilson N, Kariisa M, Seth P, Smith HT, Davis NL. Drug and opioid-involved overdose deaths: United States, 2017-2018. MMWR Morb Mortal Wkly Rep. 2020;69(11):290-7.
5. Gomes T, Tadrous M, Mamdani MM, Paterson JM, Juurlink DN. The burden of opioid-related mortality in the United States. JAMA Netw Open. 2018;1(2):e180217.

6. Jalal H, Buchanich JM, Sinclair DR, Roberts MS, Burke DS. Age and generational patterns of overdose death risk from opioids and other drugs. Nat Med. 2020;26(5):699-704.

7. Jalal H, Buchanich JM, Roberts MS, Balmert LC, Zhang K, Burke DS. Changing dynamics of the drug overdose epidemic in the United States from 1979 through 2016. Science. 2018;361(6408):eaau1184.

8. Tedesco D, Asch SM, Curtin C, Hah J, McDonald KM, Fantini MP, et al. Opioid abuse and poisoning: trends in inpatient and emergency department discharges. Health Aff (Millwood). 2017;36(10):1748-53.

9. Hasegawa K, Espinola JA, Brown DF, Camargo CA Jr. Trends in U.S. emergency department visits for opioid overdose, 19932010. Pain Med. 2014;15(10):1765-70.

10. Gaither JR, Shabanova V, Leventhal JM. US national trends in pediatric deaths from prescription and illicit opioids, 1999-2016. JAMA Netw Open. 2018;1(8):e186558.

11. Burton BN, Lin TC, Said ET, Gabriel RA. National trends and factors associated with inpatient mortality in adult patients with opioid overdose. Anesth Analg. 2019;128(1):152-60.

12. Fox LM, Hoffman RS, Vlahov D, Manini AF. Risk factors for severe respiratory depression from prescription opioid overdose. Addiction. 2018;113(1):59-66.

13. Lo-Ciganic WH, Huang JL, Zhang HH, Weiss JC, Wu YH, Kwoh $\mathrm{CK}$, et al. Evaluation of machine-learning algorithms for predicting opioid overdose risk among Medicare beneficiaries with opioid prescriptions. JAMA Netw Open. 2019;2(3):e190968.

14. Nicol AL, Colquhoun DA, Brummett CM. The more you know: identifying factors associated with inpatient mortality related to opioid overdose can drive progress in the opioid health crisis. Anesth Analg. 2019;128(1):16-8.

15. Dowell D, Zhang K, Noonan RK, Hockenberry JM. Mandatory provider review and pain clinic laws reduce the amounts of opioids prescribed and overdose death rates. Health Aff (Millwood). 2016;35(10):1876-83.

16. Rubin R. CMS to improve drug programs and opioid overuse oversight. JAMA. 2018;319(12):1189.

17. Dowell D, Haegerich TM, Chou R. CDC guideline for prescribing opioids for chronic pain: United States, 2016. JAMA. 2016;315(15):1624-45.

18. Herzig SJ, Rothberg MB, Cheung M, Ngo LH, Marcantonio ER. Opioid utilization and opioid-related adverse events in nonsurgical patients in US Hospitals. J Hosp Med. 2014;9(2):73-81.

19. St Sauver JL, Warner DO, Yawn BP, Jacobson DJ, McGree ME, Pankratz JJ, et al. Why patients visit their doctors: assessing the most prevalent conditions in a defined American population. Mayo Clin Proc. 2013;88(1):56-67.

20. DeWire SM, Yamashita DS, Rominger DH, Liu G, Cowan CL, Graczyk TM, et al. AG protein-biased ligand at the $\mu$-opioid receptor is potently analgesic with reduced gastrointestinal and respiratory dysfunction compared with morphine. J Pharmacol Exp Ther. 2013;344(3):708-17.

21. Kieffer BL. Drug discovery: designing the ideal opioid. Nature. 2016;537(7619):170-1.

22. Manglik A, Lin H, Aryal DK, McCorvy JD, Dengler D, Corder G, et al. Structure-based discovery of opioid analgesics with reduced side effects. Nature. 2016;537(7619):185-90.

23. Madariaga-Mazon A, Marmolejo-Valencia AF, Li Y, Toll L, Houghten RA, Martinez-Mayorga K. Mu-opioid receptor biased ligands: a safer and painless discovery of analgesics? Drug Discov Today. 2017;22(11):1719-29. 
24. Yekkirala AS, Roberson DP, Bean BP, Woolf CJ. Breaking barriers to novel analgesic drug development. Nat Rev Drug Discov. 2017;16(11):810.

25. Machelska H, Celik MO. Advances in achieving opioid analgesia without side effects. Front Pharmacol. 2018;29(9):1388.

26. Ding HP, Kiguchi N, Yasuda D, Daga PR, Polgar WE, Lu JJ, et al. A bifunctional nociceptin and mu opioid receptor agonist is analgesic without opioid side effects in nonhuman primates. Sci Transl Med. 2018;10(456):eaar3483.

27. Miyazaki T, Choi IY, Rubas W, Anand NK, Ali C, Evans J, et al. NKTR-181: a novel mu-opioid analgesic with inherently low abuse potential. J Pharmacol Exp Ther. 2017;363(1):104-13.

28. Dowden H, Munro J. Trends in clinical success rates and therapeutic focus. Nat Rev Drug Discov. 2019;18(7):495-6.

29. Hughes JP, Rees S, Kalindjian SB, Philpott KL. Principles of early drug discovery. Br J Pharmacol. 2011;162(6):1239-49.

30. Singh G, Schulthess D, Hughes N, Vannieuwenhuyse B, Kalra D. Real world big data for clinical research and drug development. Drug Discov Today. 2018;23(3):652-60.

31. Wise J, Moller A, Christie D, Kalra D, Brodsky E, Georgieva E, et al. The positive impacts of real-world data on the challenges facing the evolution of biopharma. Drug Discov Today. 2018;23(4):788-801.

32. Cepeda MS, Kern DM, Seabrook GR, Lovestone S. Comprehensive real-world assessment of marketed medications to guide Parkinson's drug discovery. Clin Drug Investig. 2019;39(11):1067-75.

33. Wyatt PG, Gilbert IH, Read KD, Fairlamb AH. Target validation: linking target and chemical properties to desired product profile. Curr Top Med Chem. 2011;11(10):1275-83.

34. DeShazo JP, Hoffman MA. A comparison of a multistate inpatient EHR database to the HCUP nationwide inpatient sample. BMC Health Serv Res. 2015;15(15):384.

35. Dong X, Deng J, Hou W, Rashidian S, Abell-Hart K, Rosenthal $\mathrm{RN}$, et al. Predicting opioid overdose risk of patients with opioid prescriptions using electronic health records based on temporal deep learning. J Biomed Inform. 2021;116:103725.

36. Dunn KM, Saunders KW, Rutter CM, Banta-Green CJ, Merrill JO, Sullivan MD, et al. Opioid prescriptions for chronic pain and overdose: a cohort study. Ann Intern Med. 2010;152(2):85-92.

37. Bramer GR. International statistical classification of diseases and related health problems: tenth revision. World Health Stat Q. 1988;41(1):32-6.

38. Wishart DS, Feunang YD, Guo AC, Lo EJ, Marcu A, Grant JR, et al. DrugBank 5.0: a major update to the DrugBank database for 2018. Nucleic Acids Res. 2018;46(D1):D1074-82.

39. Yu CC, Yuan M, Yang HY, Zhuang XM, Li H. P-glycoprotein on blood-brain barrier plays a vital role in fentanyl brain exposure and respiratory toxicity in rats. Toxicol Sci. 2018;164(1):353-62.

40. Smith HS. Opioid metabolism. Mayo Clin Proc. 2009;84(7):613-24.

41. Feng XQ, Zhu LL, Zhou Q. Opioid analgesics-related pharmacokinetic drug interactions: from the perspectives of evidence based on randomized controlled trials and clinical risk management. J Pain Res. 2017;10:1225-39.

42. Hingne PM, Sluka KA. Blockade of NMDA receptors prevents analgesic tolerance to repeated transcutaneous electrical nerve stimulation (TENS) in rats. J Pain. 2008;9(3):217-25.

43. Rankovic Z. CNS drug design: balancing physicochemical properties for optimal brain exposure. J Med Chem. 2015;58(6):2584-608.

44. Wager TT, Chandrasekaran RY, Hou XJ, Troutman MD, Verhoest PR, Villalobos A, et al. Defining desirable central nervous system drug space through the alignment of molecular properties, in vitro ADME, and safety attributes. Acs Chem Neurosci. 2010;1(6):420-34.

45. Quartey G, Wang J, Kim J. A review of risk measures in pharmacoepidemiology with tips for statisticians in the pharmaceutical industry. Pharm Stat. 2011;10(6):548-53.
46. Chen HN, Cohen P, Chen S. How big is a big odds ratio? Interpreting the magnitudes of odds ratios in epidemiological studies. Commun Stat Simul Comput. 2010;39(4):860-4.

47. Edlund MJ, Martin BC, Russo JE, DeVries A, Braden JB, Sullivan MD. The role of opioid prescription in incident opioid abuse and dependence among individuals with chronic noncancer pain: the role of opioid prescription. Clin J Pain. 2014;30(7):557-64.

48. Volkow ND, Frieden TR, Hyde PS, Cha SS. Medication-assisted therapies: tackling the opioid-overdose epidemic. N Engl J Med. 2014;370(22):2063-6.

49. Camilleri M, Lembo A, Katzka DA. Opioids in gastroenterology: treating adverse effects and creating therapeutic benefits. Clin Gastroenterol Hepatol. 2017;15(9):1338-49.

50. Chaves C, Remiao F, Cisternino S, Decleves X. Opioids and the blood-brain barrier: a dynamic interaction with consequences on drug disposition in brain. Curr Neuropharmacol. 2017;15(8):1156-73.

51. Morgan MM, Christie MJ. Analysis of opioid efficacy, tolerance, addiction and dependence from cell culture to human. Br J Pharmacol. 2011;164(4):1322-34.

52. Danovitch I, Vanle B, Van Groningen N, Ishak W, Nuckols T. Opioid overdose in the hospital setting: a systematic review. J Addict Med. 2020;14(1):39-47.

53. Altekruse SF, Cosgrove CM, Altekruse WC, Jenkins RA, Blanco C. Socioeconomic risk factors for fatal opioid overdoses in the United States: findings from the Mortality Disparities in American Communities Study (MDAC). PLoS ONE. 2020;15(1):e0227966.

54. West NA, Severtson SG, Green JL, Dart RC. Trends in abuse and misuse of prescription opioids among older adults. Drug Alcohol Depend. 2015;1(149):117-21.

55. Dasgupta N, Funk MJ, Proescholdbell S, Hirsch A, Ribisl KM, Marshall S. Cohort study of the impact of high-dose opioid analgesics on overdose mortality. Pain Med. 2016;17(1):85-98.

56. Volkow ND, McLellan AT. Opioid abuse in chronic pain: misconceptions and mitigation strategies. N Engl J Med. 2016;374(13):1253-63.

57. Chua KP, Brummett CM, Conti RM, Bohnert A. Association of opioid prescribing patterns with prescription opioid overdose in adolescents and young adults. JAMA Pediatr. 2020;174(2):141-8.

58. Chen Q, Larochelle MR, Weaver DT, Lietz AP, Mueller PP, Mercaldo $\mathrm{S}$, et al. Prevention of prescription opioid misuse and projected overdose deaths in the United States. JAMA Netw Open. 2019;2(2):e187621.

59. Schnoll SH. Misconceptions and realities of the prescription opioid epidemic. Clin Pharmacol Ther. 2018;103(6):963-5.

60. Fudin J, Pratt Cleary J, Schatman ME. The MEDD myth: the impact of pseudoscience on pain research and prescribing-guideline development. J Pain Res. 2016;9:153-6.

61. Schaefer CP, Tome ME, Davis TP. The opioid epidemic: a central role for the blood brain barrier in opioid analgesia and abuse. Fluids Barriers CNS. 2017;14(1):32.

62. Drewes AM, Jensen RD, Nielsen LM, Droney J, Christrup LL, Arendt-Nielsen L, et al. Differences between opioids: pharmacological, experimental, clinical and economical perspectives. Br J Clin Pharmacol. 2013;75(1):60-78.

63. Pasternak GW, Pan YX. Mu opioids and their receptors: evolution of a concept. Pharmacol Rev. 2013;65(4):1257-317.

64. Chen H, Engkvist O, Wang Y, Olivecrona M, Blaschke T. The rise of deep learning in drug discovery. Drug Discov Today. 2018;23(6):124-50.

65. Deng J, Yang Z, Li Y, Samaras D, Wang F. Towards better opioid antagonists using deep reinforcement learning. arXiv. 2020;2020:200404768.

66. Green CA, Perrin NA, Janoff SL, Campbell CI, Chilcoat HD, Coplan PM. Assessing the accuracy of opioid overdose and poisoning codes in diagnostic information from electronic health records, 
claims data, and death records. Pharmacoepidemiol Drug Saf. 2017;26(5):509-17.

67. Wei WQ, Teixeira PL, Mo H, Cronin RM, Warner JL, Denny JC. Combining billing codes, clinical notes, and medications from electronic health records provides superior phenotyping performance. $\mathrm{J}$ Am Med Inform Assoc. 2016;23(E1):E20-7.

68. Heslin KC, Owens PL, Karaca Z, Barrett ML, Moore BJ, Elixhauser A. Trends in opioid-related inpatient stays shifted after the
US transitioned to ICD-10-CM diagnosis coding in 2015. Med Care. 2017;55(11):918-23.

69. Dilokthornsakul P, Moore G, Campbell JD, Lodge R, Traugott C, Zerzan J, et al. Risk factors of prescription opioid overdose among Colorado Medicaid beneficiaries. J Pain. 2016;17(4):436-43.

70. Hah JM, Bateman BT, Ratliff J, Curtin C, Sun E. Chronic opioid use after surgery: implications for perioperative management in the face of the opioid epidemic. Anesth Analg. 2017;125(5):1733-40. 